\title{
Fetal Lamb Ventricles Respond Differently to Filling and Arterial Pressures and to in Utero Ventilation
}

\author{
MARK D. RELLER, MARK J. MORTON, DEBORAH L. REID, AND KENT L. THORNBURG \\ Departments of Pediatrics, Medicine, and Physiology. Oregon Health Sciences University. \\ Portland, Oregon 97201
}

\begin{abstract}
Right and left ventricular function were investigated in 12 fetal lambs (127-140 days gestation) instrumented with electromagnetic flow sensors on the ascending aorta and the main pulmonary artery, and with vascular catheters. Nine fetuses were equipped with a postductal aortic occluder and the trachea was cannulated in eight. Control arterial blood values were $\mathrm{pH} 7.36 \pm 0.02$ $(\mathrm{SD}), \mathrm{PCO}_{2} 49.3 \pm 2.3$ torr, $\mathrm{PO}_{2} 18.4 \pm 1.7$ torr, and hematocrit $37.3 \pm 4.4 \%$. Biventricular function curves relating stroke volume to mean right and left atrial pressure were generated by rapid withdrawal and reinfusion of fetal blood. Both function curves were composed of steep ascending and plateau limbs that intersected at a breakpoint. Stroke volumes at the breakpoints were $0.94 \pm 0.19 \mathrm{ml}$. $\mathrm{kg}^{-1}$ and $0.63 \pm 0.15 \mathrm{ml} \cdot \mathrm{kg}^{-1}$ for right and left ventricle, respectively $(p<0.001)$. During postductal aortic occlusion, arterial pressure increased by $19.3 \pm 7.9$ torr while right ventricular stroke volume decreased by $\sim 48 \%$ and left ventricular stroke volume decreased by $\sim 9 \%$. In utero ventilation increased arterial pressure, heart rate, $\mathrm{PO}_{2}$, and oxygen content. Right atrial pressure increased from $3.9 \pm$ 1.3 to $5.8 \pm 2.9$ torr $(p<0.05)$; left atrial pressure from $3.5 \pm 1.5$ to $10.0 \pm 4.4$ torr $(p<0.05)$. Aortic flow nearly doubled $\left(112 \pm 29\right.$ to $\left.211 \pm 35 \mathrm{ml} \cdot \mathrm{min}^{-1} \cdot \mathrm{kg}^{-1}\right)(p<0.05)$, and the left ventricular function curve shifted upward. The right ventricular function curve was shifted downward during ventilation. We conclude that the fetal ventricles differ significantly in their outputs, response to changes in arterial pressure, and to the onset of in utero ventilation. (Pediatr Res 22: 621-626, 1987)
\end{abstract}

From early investigations on acutely instrumented, anesthetized animals, it was concluded that the fetal ventricles have similar outputs (1). Subsequent studies in chronically instrumented, unanesthetized fetal lambs have shown a consistent right ventricular dominance under control conditions with the right ventricular output comprising $55-67 \%$ of the combined fetal cardiac output $(2,3)$. Fetal right and left ventricular stroke volumes have been measured separately during changes in filling pressure produced by hemorrhage and crystalloid infusion, and during changes in arterial pressure $(4,5)$. These studies have shown that the right ventricular function curve relating stroke volume to atrial pressure has a shape similar to the left ventricular

Received March 9, 1987; accepted July 16, 1987

Correspondence Kent L. Thornburg, Ph.D., Department of Physiology, L334, Oregon Health Sciences University, 3181 S.W. Sam Jackson Park Road, Portland, OR 97201.

Supported by USPHS HL 29324, American Heart Association/Oregon Affiliate, and the Medical Research Foundation of Oregon. function curve, but differs in that it is positioned "above" the left ventricular curve. Although these experiments support the concept of right ventricular dominance, statistical significance between the positions of the function curves was not achieved possibly because of biologic variability between animals.

When assessing the response to changes in arterial pressure. the two ventricles have been found to differ significantly $(4,5)$. In the arterial pressure range from 20-90 torr, right ventricular stroke volume was very negatively influenced by arterial pressure. while left ventricular stroke volume was minimally affected. These preliminary findings indicate that the two fetal ventricles distinctly differ in their response to changes in arterial pressure.

The two ventricles may also differ in their acute responses to the changes that take place at birth. At the time of birth, the fetal left ventricle nearly doubles its stroke volume (6). This finding has also been demonstrated during in utero ventilation (7). Mechanisms for the increased stroke volume at birth have not been conclusively shown $(6,7)$. The increase in left ventricular stroke volume with in utero ventilation occurs despite $\beta$-adrenergic blockade, and is associated with an upward shift of the left ventricular function curve, and the establishment of a left-right interatrial pressure gradient (7). As yet, right ventricular function has not been assessed during in utero ventilation. A similar upward shift in the right ventricular function curve with in utero ventilation would support the concept of a circulating inotropic substance as the cause for stroke volume increases at birth. Thus, the purpose of this investigation was to measure right and left ventricular stroke volumes simultaneously during changes in filling pressure, arterial pressure and during in utero ventilation.

\section{MATERIALS AND METHODS}

Animals. Ewes of mixed breeds were bred at our institutional farm to provide dated pregnancy and were brought to the laboratory pens several days before surgery to become accustomed to the surroundings. Fetuses of known gestation (123-133 days) were selected for instrumentation.

Surgical procedures. Ewes and fetuses underwent sterile surgery using protocols previously described $(4,5,7)$. Anesthesia was induced with intravenous methohexitol and maintained with $1 \%$ halothane in nitrous oxide and oxygen. The fetus was delivered through a midline laparotomy and hysterotomy. Polyvinyl catheters (V-5, 1.3 mm OD or V-8 1.7 mm OD, Bolab Inc., Lake Havasu City, AZ) were placed in the right carotid artery and advanced to the brachiocephalic artery and in the right jugular vein and advanced to the right atrium. The trachea was entered near the level of the thyroid gland and a double lumen $6.0 \mathrm{~mm}$ polyvinyl tubc (Argyle Salem Sump. Sherwood Medical. St. Louis, MO) was secured by circumferential suture with the tip of the tubing just above the tracheal bifurcation.

A left thoracotomy was performed in the third intercostal 
space to gain access to the fetal heart. The pericardium was opened along the pulmonary artery and from the superior vena cava on the right to the phrenic nerve on the left. The main pulmonary artery and ascending aorta were dissected free and snug fitting electromagnetic flow sensors (C \& C Instruments, Culver City, CA) were placed on the ascending aorta just above the coronary arteries and the main pulmonary artery proximal to the bifurcation of the artery and the ductus arteriosus. A V-5 catheter with a $4 \mathrm{~mm} \mathrm{V-8} \mathrm{tip} \mathrm{was} \mathrm{placed} \mathrm{in} \mathrm{the} \mathrm{left} \mathrm{atrium} \mathrm{and}$ a $3-\mathrm{cm}$ silicone rubber catheter with side holes was spliced to a long polyvinyl catheter and placed in the pericardial sac for measurement of pericardial pressure. The pericardial incision was left open. An occluder (In Vivo Metric, Heraldsburg, CA) was placed on the juxtaductal descending aorta distal to the aortic isthmus. The thoracotomy was closed in layers and a 3.3$\mathrm{mm}$ double lumen tube (Argyle Salem, Sump Sherwood Medical, St. Louis, MO) was attached to the fetal skin. The fetus was returned to the uterus and the uterus closed. All catheters, flow sensor cables, and ventilation tubing were tunneled to the side of the ewe where they were kept in nylon pouches. The tracheal and smaller Salem sump tube were joined in a closed loop to allow for fetal pulmonary drainage. Penicillin $\left(10^{6} \mathrm{U}\right)$ was introduced into the amniotic cavity at the end of the procedure. Following surgery, ewes were brought to a clean pen where they recovered from surgery for several days before experiments were performed.

Laboratory procedures. On the day of the experiment, the ewe was put into a stanchion and given food and water ad libidum. Hydrostatic pressures were converted to electrical signals by I.D. Statham Gould (Gould Inc., Oxnard, CA) pressure transducers. Pressures were measured from the right atrium (via the jugular vein), left atrium, aortic arch (via the carotid artery), trachea, amniotic and pericardial spaces, and were recorded on a Beckman R611 recorder (Beckman Instruments, Schiller Park, IL). Transducers were calibrated to the nearest 0.5 torr with a water manometer. All vascular pressures were referenced to the pericardial pressure. The flow sensors were calibrated preoperatively in vitro using appropriate caliber sheep arteries and blood. The flow meters (Gould SP2202, Gould Inc.) were adjusted to zero during diastole when aortic and pulmonary arterial flow were assumed to be zero.

Outputs from all channels were recorded onto floppy disks utilizing a Hewlett-Packard 3437A system voltmeter and 9826s computer (Desk Top Computer Division, Fort Collins, CO). Digital signals were sampled every $10 \mathrm{~ms}$ and averaged every 5 s. All output data from the disk were checked against original strip chart records.

Experimental protocol. With vascular catheters opened, but prior to other manipulations, arterial blood gas tensions, $\mathrm{pH}$, and oxygen contents were measured. Animals with an arterial $\mathrm{PO}_{2}$ greater than or equal to 16 torr and $\mathrm{pH}$ greater than or equal to 7.3 during the control period were accepted for the protocol. Control values of vascular pressures, simultaneous left and right ventricular outputs, and heart rate were recorded. Fetuses were given the cholinergic blocking agent, atropine $(0.5 \mathrm{mg} / \mathrm{kg})$, and the $\beta$-adrenergic blocking agent, propranolol $(1 \mathrm{mg} / \mathrm{kg})$, to minimize the effects of autonomic compensatory mechanisms (8). A second set of hemodynamic values were recorded during cardiac autonomic blockade. The fetuses were then studied under three protocols to determine the responses of the right and left ventricular stroke volume to changes in filling and arterial pressures and to in utero ventilation.

Protocol $A$. Left and right ventricular performances were studied simultaneously by generating function curves relating left and right ventricular outputs measured by electromagnetic flow sensors to mean left and right atrial pressures, respectively. Biventricular function curves were formed by rapidly withdrawing and reinfusing fetal blood through a V-8 carotid artery catheter as previously described $(4,5,7)$. A plot of right and left ventricular stroke volumes as a function of the respective mean atrial pressures was observed on the cathode ray tube of the microcomputer to vary atrial pressure between 1 and 8 torr.

Protocol B. Following a recovery period of 15-30 min, cardiac autonomic blockade was readministered and the simultaneous responses of left and right ventricular outputs to increasing arterial pressure were studied. If necessary, the filling pressure was increased by bolus infusion of Ringer solution until the plateau of the function curve was reached. Arterial pressure was increased by stepwise increments of inflation of the juxtaductal aortic occluder. Arterial pressure was increased about 20 torr and then the occluder was released. The stepwise occlusion and release were completed in $60 \mathrm{~s}$. Ventricular outputs and arterial pressure were recorded continuously and a plot of right and left ventricular stroke volume as a function of arterial pressure was monitored on the cathode ray tube of the microcomputer.

Protocol $C$. The tracheal tube was connected to a respiratory pump (Harvard Apparatus Co., model 607a, South Natick, MA) and the fetus was ventilated with oxygen as previously described $(7,9)$. The fetus was respired at a ventilatory rate of $40-50 / \mathrm{min}$ and tidal volumes of $15-25 \mathrm{ml} \cdot \mathrm{kg}^{-1}$ estimated fetal weight. Peak inspiratory tracheal pressures were between 15 and 25 torr. After a 15- to 20-min ventilation period, cardiac autonomic blockade was readministered and control hemodynamic data were obtained. Arterial blood was obtained for measurement of $\mathrm{pH}$, gas tensions, and oxygen content. Following these control measurements and during ventilation, left and right ventricular performance were studied in a manner identical to protocol A except for the maintenance of pulmonary ventilation with oxygen. At the end of experimentation, the ewe and fetus were killed with an intravenous bolus injection of barbiturate. The fetus was weighed and autopsied to determine catheter positions, flow probe fit, and healing.

\section{STATISTICAL ANALYSIS}

Function curves relating stroke volume to mean atrial pressure were subjected to analysis by least squares of two intersecting lines $(10,11)$. The best intersection point (breakpoint) was determined from the average of the seven atrial pressures which gave the lowest sum of residuals (5). The function curve consisted of an ascending limb, breakpoint, and a plateau limb, each characterized mathetmatically. Function curves were compared by two methods: one-way analysis of variance for the regression coefficients and breakpoints, or by two-way analysis of covariance on data normalized to control breakpoint values of stroke volume and atrial pressures $(7,12)$.

\section{RESULTS}

Successful experiments were performed in 12 fetal lambs. Gestational ages, weights, days postoperation, and the protocols performed are indicated for each fetus in Table 1. Control arterial blood gases and oxygen contents and their responses to pulmonary ventilation are shown in Table 2 . Control hemodynamic data before and after cardiac autonomic blockade and during pulmonary ventilation are shown in Table 3 . Left ventricular stroke volumes are not corrected for coronary flow.

Protocol $A$. Simultaneous left and right ventricular function curves were generated prior to ventilation in all 12 fetuses. The simultaneous right and left ventricular function curves for fetus 10 and the average function curves for all 12 fetuses are shown in Figure 1. The curves of the two ventricles were similar in shape in that with increasing atrial pressure, ventricular stroke volume increased rapidly until a mean filling pressure of approximately 3-4 torr was reached as shown previously $(4,5)$. Increasing filling pressures of more than 3-4 torr was associated with only minimal further increases in ventricular stroke volume. The left and right ventricular function curves differed in that for any given filling pressure, right ventricular stroke volume exceeded left ventricular stoke volume (Fig. 1). The stroke volumes and 
atrial pressures at the breakpoint (the intersection between the ascending and plateau limbs) for the left and right ventricle did not differ from the stroke volumes and atrial pressure noted in the control state prior to the experimental manipulation (Table $3)$. The slopes of the ascending limbs of the right and left ventricular function curves were not different $(p=0.93)$. The slope of the plateau limb of the left ventricular function curve was greater than the plateau of the right ventricular function curve at a borderline significance level $(p=0.06)$.

Protocol $B$. In nine of the fetal lambs, we were able to measure the changes in ventricular stroke volumes during increases in arterial pressure produced by occlusion of the descending aorta. During occlusion, arterial pressure increased from $58.5 \pm 6.5$ to $78.7 \pm 9.7$ torr. Both ventricular stroke volumes decreased during aortic occlusion; however, the decrement in ventricular output per unit increase in arterial pressure was almost 5-fold greater for the right ventricle than for the left $(p<0.001)$ (Fig. 2).

Protocol $C$. Eight fetal lambs were ventilated with oxygen in

Table 1. Experimental animals and protocols

\begin{tabular}{ccccl}
\hline Fetus & $\begin{array}{c}\text { Gestational age } \\
\text { (days) }\end{array}$ & Wt (kg) & $\begin{array}{c}\text { Days } \\
\text { postoperation }\end{array}$ & $\begin{array}{c}\text { Experimental } \\
\text { protocols* }\end{array}$ \\
\hline 1 & 127 & 2.0 & 4 & A \\
2 & 132 & 3.9 & 5 & A, B, C \\
3 & 137 & 3.5 & 6 & A, B \\
4 & 139 & 5.0 & 6 & A, B, C \\
5 & 138 & 4.6 & 6 & A, C \\
6 & 140 & 3.8 & 11 & A, B \\
7 & 139 & 3.0 & 9 & A \\
8 & 139 & 3.4 & 10 & A, B, C \\
9 & 140 & 6.0 & 10 & A, B, C \\
10 & 139 & 4.3 & 7 & A, B, C \\
11 & 140 & 5.4 & 10 & A, B, C \\
12 & 138 & 3.5 & 8 & A, B, C \\
& & & & \\
Mean & 137 & 4.0 & 7.7 & A, $n=12$ \\
\pm SD & & & & B, $n=9$ \\
\hline
\end{tabular}

* Protocol $\mathrm{A}=$ left and right ventricular function curves relating stroke volume to mean atrial pressure were generated simultaneously in the control state. Protocol $\mathrm{B}=$ simultaneous response of left and right ventricular stroke volumes to increases in arterial pressure. Protocol $\mathrm{C}=$ in utero ventilation with generation of simultaneous left and right ventricular function curves. utero. Ventilation resulted in a doubling of the fetal arterial oxygen content with a corresponding increase in $\mathrm{PO}_{2}$ (Table 2). The hemodynamic responses to in utero ventilation are shown in Table 3. Figure 3 shows the time course of hemodynamic changes after the initiation of in utero ventilation in a representative fetus. Simultaneous left and right ventricular function curves were obtained during in utero ventilation as described in protocol A. Average function curves and breakpoints before and after ventilation are shown in Figure 4 . The left ventricular function curve was shifted upward and the breakpoint left atrial pressure increased from $2.3 \pm 0.8$ to $4.5 \pm 1.1$ torr $(p<0.001)$. Neither the slope of the ascending nor plateau limb was changed by ventilation. Breakpoint left ventricular stroke volume was increased by $70 \%(p<0.001)$. In contrast, the ascending and plateau limbs of the right ventricular function curve was shifted downward $(p<0.005)$ during in utero ventilation with oxygen. The slopes of the limbs were unchanged.

\section{DISCUSSION}

We believe this study accurately defines the responses of the mature fetal lamb ventricles to changing hemodynamic conditions and in utero ventilation. The fetuses were studied unanesthetized and remote from the trauma of surgical instrumentation. Arterial blood gas measurements, $\mathrm{pH}$, and hematocrit were similar to measurements obtained in other studies of chronically instrumented fetal lambs $(2,4,5,13)$. The control measurements of hemodynamic values are similar to values reported from this laboratory and others for atrial and arterial pressures and for heart rate in chronically instrumented fetal lambs. The right and left ventricular stroke volume values are lower in this report than we have noted in separate studies of the ventricles using flow probes $(4,5)$ or that others have noted using indicator dilution techniques $(2,3)$. We are unable to explain this discrepancy. The flow probes ( $C \& C$ Intruments) were similar to those used for previous experiments and were calibrated in the identical manner with sheep blood and excised sheep blood vessels. We note that the responses of the individual ventricles to filling or arterial pressure changes are consistent with previous investigations of the ventricles studied separately $(4,5)$. Furthermore, the percentage increase in left ventricular stroke volume at the breakpoint of the function curve during ventilation $(70 \%)$ is similar to the increase reported when the left ventricle was studied alone $(62 \%)$. We conclude that the reduced values of right and left ventricular output reported herein may represent normal biologic

Table 2. Arterial blood gas tensions, oxygen contents, and hematocrits in control state and following in utero ventilation

\begin{tabular}{|c|c|c|c|c|c|}
\hline & $\mathrm{pH}$ & $\mathrm{P}_{\mathrm{CO}_{2}}$ & $\mathrm{P}_{\mathrm{O}_{2}}$ & $\mathrm{CaO}_{2}$ & Hematocrit (\%) \\
\hline Control & $7.36 \pm 0.02$ & $49.3 \pm 2.3$ & $18.4 \pm 1.7$ & $6.6 \pm 1.3$ & $37.3 \pm 4.4$ \\
\hline Ventilation & $7.36 \pm 0.04$ & $41.7 \pm 4.3^{*}$ & $105.4 \pm 118.0^{*}$ & $13.4 \pm 2.3^{*}$ & $39.1 \pm 4.0$ \\
\hline
\end{tabular}

${ }^{*} p<0.05$, ventilation versus control.

Table 3. Hemodynamic values before and after cardiac autonomic blockade and during in utero ventilation (mean $\pm S D)^{*}$

\begin{tabular}{|c|c|c|c|c|c|c|c|c|}
\hline & RAP (torr) & LAP (torr) & AP (torr) & $\begin{array}{c}\mathrm{Q} \text { aorta } \\
\left(\mathrm{ml} \cdot \mathrm{min}^{-1} \cdot \mathrm{kg}^{-1}\right)\end{array}$ & $\begin{array}{l}\text { Q pulmonary } \\
\left(\mathrm{ml} \cdot \mathrm{min}^{-1} \cdot \mathrm{kg}^{-1}\right)\end{array}$ & $\begin{array}{c}\text { LVSV } \\
\left(\mathrm{ml}^{-} \cdot \mathrm{kg}^{-1}\right)\end{array}$ & $\begin{array}{c}\text { RVSV } \\
\left(\mathrm{ml} \cdot \mathrm{kg}^{-1}\right)\end{array}$ & $\begin{array}{c}\mathrm{HR} \\
\left(\mathrm{min}^{-1}\right)\end{array}$ \\
\hline Control & $3.5 \pm 0.9$ & $3.2 \pm 1.1$ & $49.2 \pm 6.7$ & $107 \pm 25$ & $160 \pm 28$ & $0.67 \pm 0.16$ & $1.02 \pm 0.25$ & $160 \pm 21$ \\
\hline Blocked $†$ & $3.9 \pm 1.3$ & $3.5 \pm 1.5$ & $50.5 \pm 5.6$ & $112 \pm 29$ & $167 \pm 33$ & $0.64 \pm 0.21$ & $0.95 \pm 0.22$ & $177 \pm 20$ \\
\hline Ventilation $\ddagger$ & $5.8 \pm 2.9$ & $10.0 \pm 4.4$ & $60.3 \pm 11.2$ & $211 \pm 35$ & $164 \pm 22$ & $1.07 \pm 0.16$ & $0.83 \pm 0.13$ & $198 \pm 17$ \\
\hline Significance & $\S \|$ & $\S \|$ & $\S \|$ & $\S \cdot \|$ & NSף & $\S \cdot \|$ & NST & $\S \cdot \|^{* * *}$ \\
\hline
\end{tabular}

* RAP, right atrial pressure; LAP, left atrial pressure; AP, arterial pressure; $\dot{Q}$ aorta, mean aortic flow; $\mathrm{Q}$ pulmonary, mean pulmonary artery flow; LVSV, left ventricular stroke volume; RVSV, right ventricular stroke volume; HR, heart rate.

+ Values obtained after $\beta$-adrenergic and cholinergic blockade.

$\$$ Values obtained after $\beta$-adrenergic and cholinergic blockade and during in utero pulmonary ventilation with oxygen.

$\S$ control versus ventilation different $p<0.005$.

$\|$ blocked versus ventilation different $p<0.05$.

I No significant difference among or between groups.

** control versus blocked different $p<0.05$. 

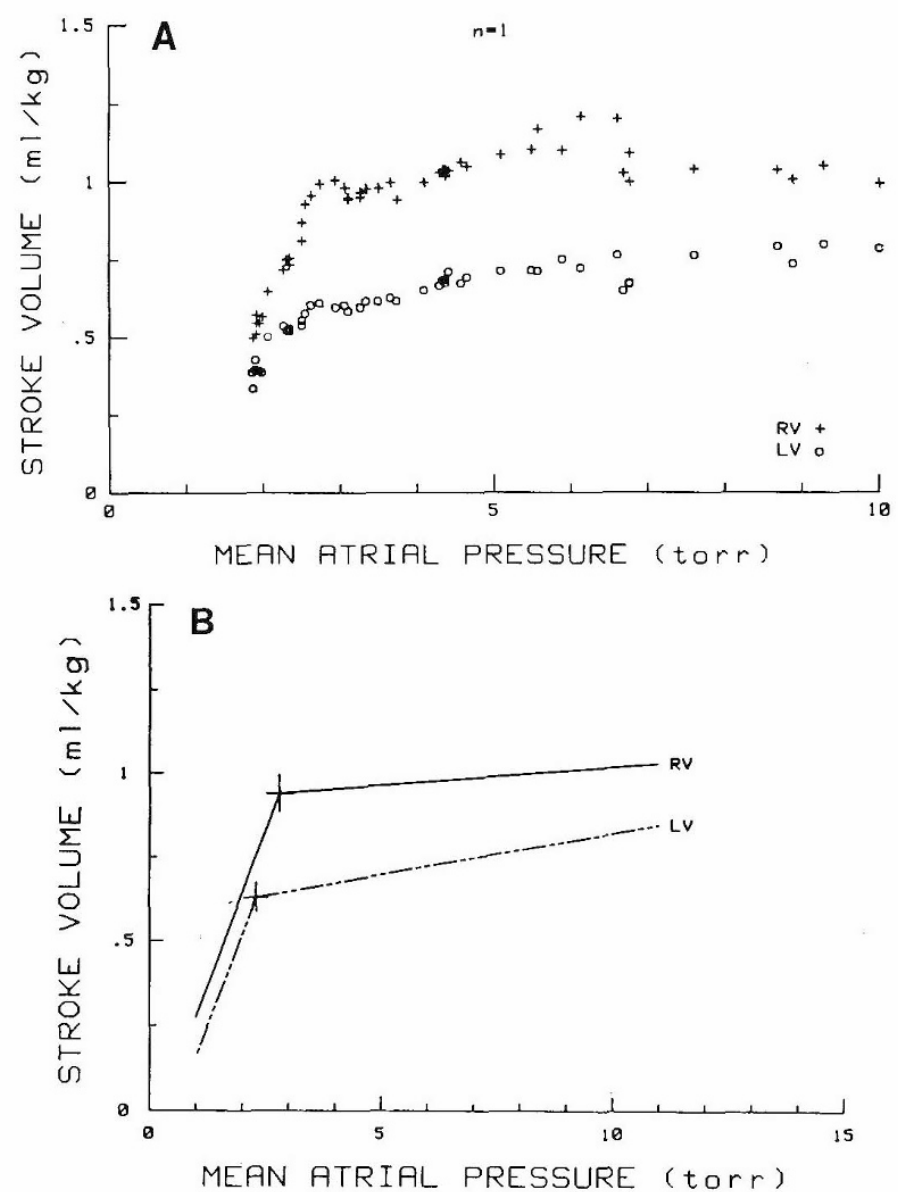

Fig. 1. $A$, simultaneous stroke volumes are shown for the right ventricle (+) and left ventricle ( $\square$ ) of a fetus during alterations of mean right and left atrial pressures produced by rapid hemorrhage and reinfusion. Each data point represents a 5-s average. Each function curve consists of a steep ascending limb at low atrial pressures and a plateau limb at elevated atrial pressures. $B$, the average simultaneous function curves for all 12 fetuses were determined by forcing the average regression coefficients for the ascending and plateau limbs of the right and left ventricular function curves through their respective average breakpoint stroke volume-mean atrial pressure coordinates. The right ventricular function curve has a steep ascending limb with a mean slope of $+0.37 \pm 0.17 \mathrm{ml}$. $\mathrm{kg}^{-1} \cdot \mathrm{torr}^{-1}$ and a plateau limb with a mean slope of $+0.01 \pm 0.02 \mathrm{ml}$. $\mathrm{kg}^{-1} \cdot$ torr $^{-1}$. The stroke volume intercept of the two limbs is $0.94 \pm 0.19$ $\mathrm{ml} \cdot \mathrm{kg}^{-1} \cdot \mathrm{min}^{-1}$. The left ventricular function curve has a steep ascending limb with a mean slope of $+0.36 \pm 0.26 \mathrm{ml} \cdot \mathrm{kg}^{-1} \cdot$ torr $^{-1}$ and a plateau limb with a mean slope of $+0.03 \pm 0.02 \mathrm{ml} \cdot \mathrm{kg}^{-1} \cdot$ torr $^{-1}$. The stroke volume intercept of the two limbs is $0.63 \pm 0.15 \mathrm{ml} \cdot \mathrm{kg}^{-1}$, a value that significantly differs from the intercept right ventricular stroke volume ( $p$ $<0.001)$.

variability or may be due to an unrecognized effect of the experimental preparation. Nevertheless, the data presented are internally consistent and firmly establish the relative position of the right and left ventricular function curves before and during in utero ventilation and the differential effects of arterial pressure on right and left ventricular stroke volume.

Right ventricular dominance was described by Heymann et al. (3) in the chronically instrumented fetal lamb, but questioned in the human later by St. John Sutton et al. $(15,16)$ who studied hearts with echocardiography and by postmortem morphometry. Subsequent Doppler studies of human fetuses support the concept of right ventricular dominance in our species $(17,18)$. We performed a volumetric and morphometric analysis of the mature fetal lamb heart in order to determine whether anatomic features might explain right ventricular dominance (19). The
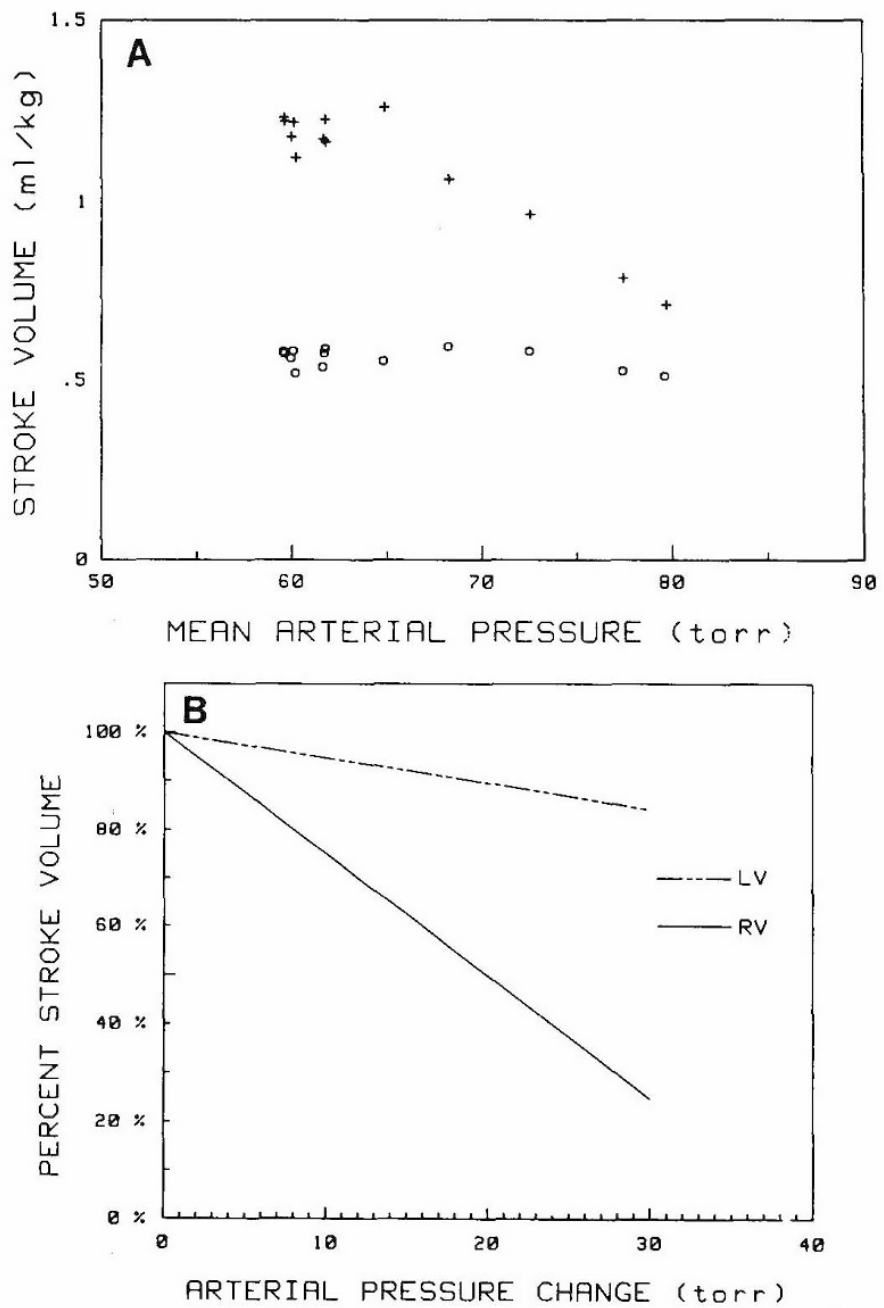

Fig. 2. $A$, simultaneous right ventricular (+) and left ventricular ( $\square$ ) stroke volumes of a fetus are shown during stepwise increases in arterial pressure produced by inflation of an occluder on the descending aorta. Each data point represents a 5-s average. Although right ventricular stroke volume is greater than left ventricular stroke volume under control conditions, right ventricular stroke volume is much more sensitive to increased arterial pressure than left ventricular stroke volume. $B$, the simultaneous average responses of the right and left ventricles to increased arterial pressure are shown for nine fetuses. Stroke volume is expressed as a percent of control value and arterial pressure as the increment above control. The linear regression coefficient for each ventricle was calculated, the average slope forced through $100 \%$ on the $Y$ axis and the lines extended through the pressure range studied. The right ventricular pressure sensitivity $\left(-2.5 \pm 1.4 \%\right.$ stroke volume $\cdot$ torr $\left.^{-1}\right)$ was more than five times the left ventricular pressure sensitivity $(-0.5 \pm$ $0.7 \%$ stroke volume $\cdot$ torr $\left.^{-1}\right)(p<0.001)$.

fetal lamb right ventricular volume is greater than left ventricular volume at all common filling pressures. Thus, if right and left ventricular ejection fractions are similar, the fetal right ventricle will have a larger stroke volume than the left ventricle at all common filling pressures and this is the result found in our study.

The shape of the fetal biventricular function curve was first described by Gilbert (20) using three points obtained by an indicator dilution technique. The steep relationship between atrial pressure and stroke volume at low filling pressures and the relatively flat relationship at atrial pressures above control are common to both the right and left ventricular function curves in the present study. It must be recognized that the function curves derived in this and previous studies $(4,5,7,20,21)$ have associated arterial pressure changes. Thus, their shape would be 


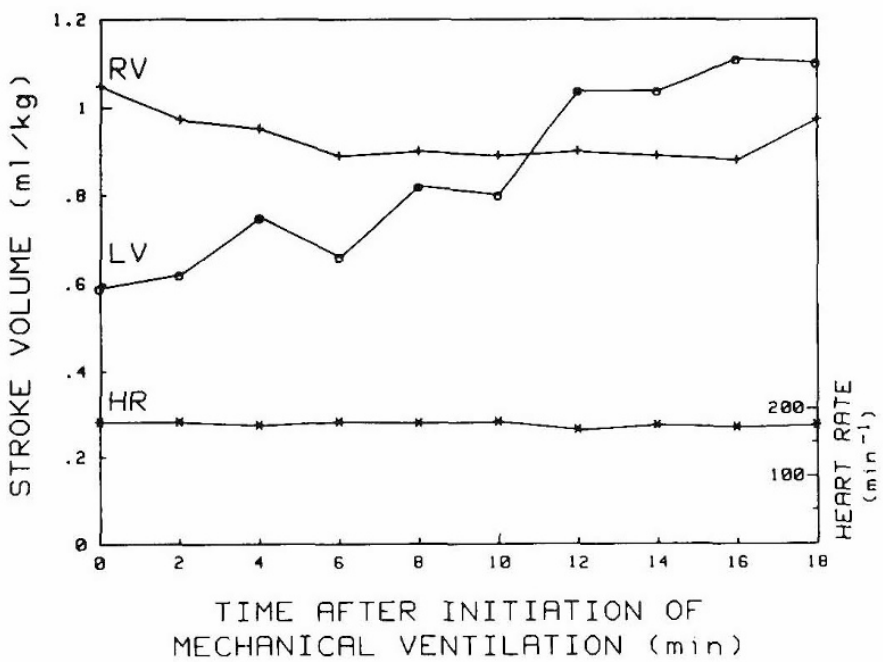

Fig. 3. The time course of right ventricular stroke volume (+), left ventricular stroke volume $(\square)$, and heart rate $(x)$ are shown for a fetus during the initiation of mechanical ventilation with oxygen in utero. The increase in left ventricular stroke volume is rapid and reaches right ventricular values within $10 \mathrm{~min}$. Right ventricular stroke volume falls slightly. Heart rate falls about 30 beats/min in the absence of cholinergic blockade. After cholinergic and $\beta$-adrenergic blockade, ventilation, on average, raised heart rate (Table 3 ).

different at constant arterial pressure with a slight increase in both the ascending and plateau slopes owing to the negative relationship between arterial pressure and stroke volume.

Gilbert (21) also described the inverse relationship between biventricular output and arterial pressure. It is now apparent that the inverse relationship between stroke volume and arterial pressure resides largely with the right ventricle. The reductions in stroke volume shown in Figure $2 B$ were accomplished by increased atrial as well as arterial pressure. Thus, although care was taken to assure that the ventricles were studied on the plateau limb of the function curve, the curves might be steeper were atrial pressure held constant. We do not believe changing atrial pressure can account for the different responses of the two ventricles. The explanation for differential sensitivity to arterial pressure may, again, be in the morphology of the fetal ventricles (19). Not only is the fetal right ventricle larger than the left ventricle, but the circumferential radius of curvature to wall thickness ratio is nearly twice as great in the right than left ventricle (19). This ratio, an important determinant of wall stress and, therefore afterload, indicates that the fetal right ventricle may have a much higher afterload at any given arterial pressure than the left ventricle. Thus, the differential effects of arterial pressure on stroke volume in the fetal ventricles described herein confirm previous reports of ventricles studied separately and are consistent with fetal ventricular morphometry. Another explanation for the differing pressure sensitivities of the fetal ventricles might lie in the impedances of the aorta and pulmonary artery. If important differences in impedance exist, a constriction distal to the ductus might have a different effect on the right than the left ventricle.

That the two ventricles have different sensitivities to arterial pressure has interesting implications in pathologic situations. Increases in systemic arterial pressure in association with fetal stress or hypoxia (22-24) would be expected to decrease right ventricular stroke volume to a much greater extent than left, a finding that might adversely affect blood flow and oxygen delivery to the lower body. The discrepancy between right and left ventricular stroke volume responses to arterial pressure might be even further pronounced in the stressed newborn with birth asphyxia and persistent hypoxemia prior to closure of the ductus arteriosus. There is ample clinical evidence of selective right
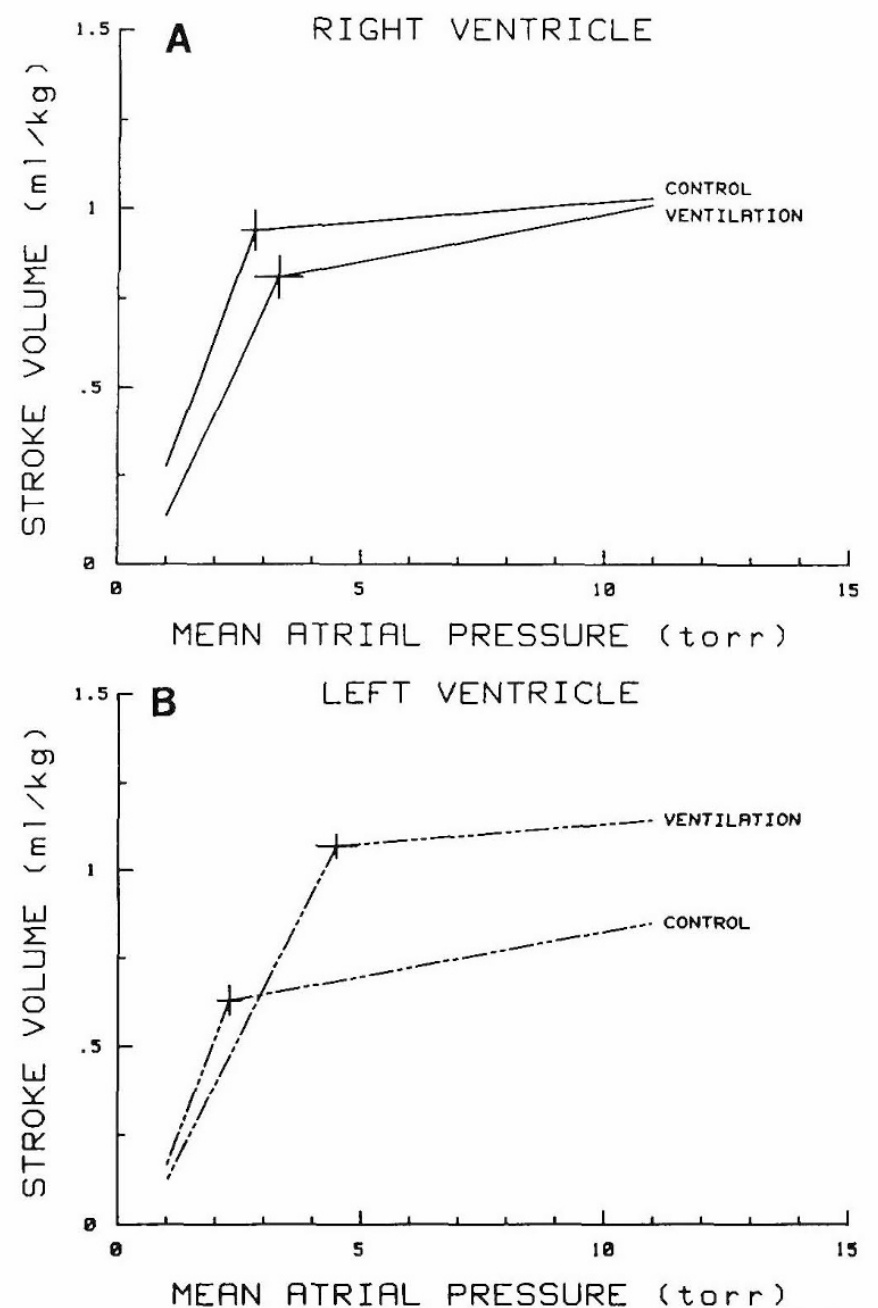

Fig. 4. Average function curves for the right and left ventricle before and during in utero ventilation were constructed as described in Figure $1(n=8)$. A, the right ventricular function curve was depressed by in utero ventilation (analysis of covariance, $p<0.005$ ). The control breakpoint stroke volume, $0.94 \pm 0.19 \mathrm{ml} \cdot \mathrm{kg}^{-1}$ at $2.8 \pm 1.0$ torr, was not different from the breakpoint during ventilation, $0.81 \pm 0.16 \mathrm{ml} \cdot \mathrm{kg}^{-1}$ at $3.3 \pm 1.3$ torr. The ascending limb slopes, $0.37 \pm 0.17$ and $0.29 \pm 0.29$ $\mathrm{ml} \cdot \mathrm{kg}^{-1} \cdot \mathrm{torr}^{-1}$, and the plateau slopes, $0.01 \pm 0.02$ and $0.03 \pm 0.02 \mathrm{ml}$. $\mathrm{kg}^{-1} \cdot$ torr $^{-1}$, were not different between control and ventilation, respectively. $B$, the left ventricular function curve was shifted upward during in utero ventilation (analysis of covariance, $p<0.001$ ). The breakpoint stroke volume increased from $0.63 \pm 0.15$ to $1.07 \pm 0.09 \mathrm{ml} \cdot \mathrm{kg}^{-1}$ while atrial pressure increased from $2.3 \pm 0.8$ to $4.5 \pm 1.1$ torr $(p<0.001)$ during ventilation. The ascending limb slopes, $0.36 \pm 0.26$ and $0.27 \pm$ $0.10 \mathrm{ml} \cdot \mathrm{kg}^{-1} \cdot \mathrm{torr}^{-1}$ and plateau limb slopes, $0.03 \pm 0.02$ and $0.01 \pm$ $0.02 \mathrm{ml} \cdot \mathrm{kg}^{-1} \cdot \mathrm{torr}^{-1}$ were not different between control and ventilation, respectively.

ventricular dysfunction in this setting of neonatal hypoxemia and persistent pulmonary hypertension of the newborn $(25,26)$.

We are uncertain as to the exact relationship between the hemodynamic adjustments at birth and those seen during in utero ventilation. Preliminary reports from Rudolph's (27-29) laboratory show that placental flow is decreased, foramenal flow nearly abolished, and ductal flow reversed during in utero ventilation. Pulmonary blood flow is dramatically increased. Thus, it appears that in utero ventilation mimics the hemodynamic events at the moment of birth. Of course, by circumventing the birth process itself and the need for neonatal thermogenesis and respiration, it is easier to examine the precise hemodynamic events that lead to increased cardiac output at birth.

The mechanisms for left ventricular stroke volume increase at 
birth remain undefined. There is no evidence for decreased left ventricular afterload either with birth or with in utero ventilation; aortic pressure is increased in both instances. Contractility might be increased by catecholamines at birth. Klopfenstein and Rudolph (8) showed previously that $\beta$-adrenergic blockade did not significantly reduce left ventricular stroke volume in the neonatal lamb. In this and our previous study (7) of left ventricular stroke volume during in utero ventilation, the increase in stroke volume occurred despite $\beta$-adrenergic blockade. We cannot exclude the possibility that oxygen is inotropic in the fetus or that some other inotropic substance is released from the lungs with ventilation. However, the careful study of Anderson et al. (30) of fetal lamb cardiac dimensions and shortening shows no important change in fractional shortening after birth despite a measureable increase in inotropy. Furthermore, both Anderson et al. (30) and Kirkpatrick et al. (31) found increased left ventricular dimension at birth. Thus, we believe that the most likely mechanism for left ventricular stroke volume increase at birth is increased left ventricular end-diastolic volume. The fact that a stroke volume increase of this magnitude cannot be brought about by increased left atrial pressure before birth $(5,7)$ suggests that the left ventricular pressure-volume relationship is altered at birth and with in utero ventilation. The left ventricular pressure-volume relationship could be shifted by ventricular interaction through the establishment of a left to right atrial pressure gradient $(32,33)$. The importance of right ventricular pressure to left ventricular volume in fetal lamb hearts has been demonstrated $(19,34)$.

How right ventricular stroke volume is increased at birth remains unanswered. We found that the right ventricular function curve was actually shifted downward with in utero ventilation. We believe that this is further evidence against a prominent role of an inotropic substance released at birth because there is no obvious reason that the right ventricle should not respond to the same extent as the left ventricle to a circulating inotropic substance. Furthermore, the fact that the right ventricular function curve shifted downward may be additional evidence for ventricular interaction as an important mechanism for changes in cardiac function at birth. As left ventricualr diastolic pressure increases with respect to right ventricular pressure, right ventricular volume should be lower at any given filling pressure. If true, how could right ventricular stroke volume go up at birth? The mechanism for the subsequent increase in right ventricular stroke volume after birth may be reduced pulmonary artery pressure and impedance. As noted previously, fetal right ventricular stroke volume is inversely related to pulmonary artery pressure (4). Thus, the anticipated upward shift of the right ventricular function curve may not be seen until pulmonary vascular resistance has completed its fall and the ductus arteriosus has closed.

Acknowledgments. The authors acknowledge the assistance of Thomas J. Green, Robert C. Webber, Dana J. McNaught, and Jacqueline Niemi.

\section{REFERENCES}

1. Dawes GS 1968 The foetal circulation. In: Foetal and Neonatal Physiology. Year Book Medical Publishers, Chicago, pp 94-95

2. Anderson DF, Bissonnette JM, Faber JJ, Thornburg KL 1981 Central shunt flows and pressures in the mature fetal lamb. Am J Physiol 241:H60-H66

3. Heymann MA, Creasy RK, Rudolph AM 1973 Quantitation of blood flow pattern in the foetal lamb in utero. In: Comline KS, Cross KW, Dawes GS, Nathanielsz PW (eds) Foetal and Neonatal Physiology. Cambridge University Press, Cambridge, England, pp 129-135

4. Thornburg KL, Morton MJ 1983 Filling and arterial pressures as determinants of RV stroke volume in the sheep fetus. Am J Physiol 244:H656-H663

5. Thornburg KL, Morton MJ 1986 Filling and arterial pressures as determinants of left ventricular stroke volume in fetal lambs. Am J Physiol 251:H961H968

6. Rudolph AM 1985 Distribution and regulation of blood flow in the fetal and neonatal lamb. Circ Res 57:811-821

7. Morton MJ, Pinson CW, Thornburg KL 1987 In utero ventilation with oxygen augments left ventricular stroke volume in lambs. J Physiol 383:413-424

8. Klopfenstein HS, Rudolph AM 1978 Postnatal changes in the circulation and responses to volume loading in sheep. Circ Res 42:839-845

9. Willis DM, Anderson DF, Thornburg KL, Faber JJ 1985 Alteration of arterial blood gas composition by positive pressure ventilation in the unanesthetized fetal lamb in utero. Biol Neonate 47:295-304

10. Wallenstein S, Zucher CL, Fleiss JL 1980 Some statistical methods useful in circulation research. Circ Res 47:1-9

11. Orr GW, Green HJ, Hughson RL Bennett GW 1982 A computer linear regression model to determine ventilatory anaerobic threshold. J Appl Physiol 52:1347-1352

12. Winer BJ 1971 Analysis of covariance. In: Statistical Principles in Experimental Design, 2nd ed. McGraw-Hill, New York, pp 752-812

13. Fisher DJ, Heymann MA, Rudolph AM 1980 Myocardial oxygen and carbohydrate consumption in fetal lambs in utero and in adult sheep. Am J Physio 238:H399-H405

14. Shinebourne EA, Vapaavouri EK, Williams RL, Heymann MA, Rudolph AM 1972 Development of baroreflex activity in unanesthetized fetal and neonatal lambs. Circ Res 31:710-718

15. St. John-Sutton MG, Raichien JS, Reichek N, Huff DS 1984 Quantitative assessment of right and left ventricular growth in the human fetal heart: a pathoanatomic study. Circulation 70:935-941

16. St. John-Sutton MG, Gewitz MH, Shah B, Cohen A, Reichek N, Gabbe S, Huff DS 1984 Quantitative assessment of growth and function in the cardiac chambers in the normal human fetus: a prospective longitudinal echocardiographic study. Circulation 69:645-654

17. Reed KL, Meijboom EJ, Sahn DJ, Seagnelli SA, Valdes-Cruz LM, Shenker L 1986 Cardiac Doppler flow velocities in human fetuses. Circulation 73:41 46

18. Kenny JF, Plappert T, Doubilet P, Saltzman DH, Cartier M, Zollars L, Leatherman GF, St John-Sutton MG 1986 Changes in intracardiac blood flow velocities and right and left ventricular stroke volumes with gestational age in the normal human fetus: a prospective Doppler echocardiographic study. Circulation 74:1208-1216

19. Pinson CW, Morton MJ, Thornburg KL 1987 An anatomic basis for fetal right ventricular dominance and arterial pressure sensitivity. J Dev Physiol 9:253271

20. Gilbert RD 1980 Control of fetal cardiac output during changes in blood volume. Am J Physiol 238:H80-H85

21. Gilbert RD 1982 Effects of afterload and baroreceptors on cardiac function in fetal lambs. J Dev Physiol 4:299-310

22. Cohn HE, Sacks EJ, Heymann MA, Rudolph AM 1974 Cardiovascular response to hypoxemia and acidemia in fetal lambs. Am J Obstet Gynecol 120:817-824

23. Cohn HE, Piasecki GJ, Jackson BT 1980 The effect of fetal heart rate on cardiovascular function during hypoxemia. Am J Obstet Gynecol 138:11901198

24. Cohen WR, Piasecki GJ, Jackson BT 1982 Plasma catecholamines during hypoxemia in fetal lambs. Am J Physiol 243:R520-R525

25. Riemenschneider TA, Nieben HC, Ruttenberg HD, Jaffe RB 1976 Disturbances of the transitional circulation: spectrum of pulmonary hypertension and myocardial dysfunction. J Pediatr 89:622-625

26. Bucciarelli R, Nelson RM, Egan EA, Eitzman DV, Gessner IH 1977 Transient tricuspid insufficiency of the newborn: a form of myocardial dysfunction in stressed newborns. Pediatrics 59:330-339

27. Rudolph AM, Teitel DF, Iwamoto HS, Gleason CA 1986 Ventilation is more important than oxygenation in reducing pulmonary vascular resistance a birth. Pediatr Res 20:439A(abstr)

28. Teitel DF, Iwamoto HS, Rudolph AM 1986 Redistribution of cardiac output during birth simulation. Pediatr Res 20:372A(abstr)

29. Iwamoto HS, Teitel DF, Rudolph AM 1986 Effects of birth events on regional blood flows. Pediatr Res 20:189A(abstr)

30. Anderson PAW, Glick KL, Manring A, Crenshaw CC, Jr 1984 Developmental changes in cardiac contractility in fetal and postnatal sheep: In vitro and in vivo. Am J Physiol 247:H371-H379

31. Kirkpatrick SE, Covell JW, Friedman WF 1973 A new technique for the continuous assessment of fetal and neonatal cardiac performance. Am J Obstet Gynecol 116:963-972

32. Glantz SA, Parmley WW 1978 Factors which affect the diastolic pressurevolume curve. Circ Res 42:171-180

33. Elzinga G, Van Grondelle R, Westerhof N, Van den Bos GC 1974 Ventricular interference. Am J Physiol 226:941-947

34. Romero T, Covell JW, Friedman WF 1972 A comparison of pressure-volume relations of the fetal, newborn, and adult heart. Am J Physiol 222:12851290 